\title{
中空 $\mathrm{SiO}_{2}$ 微球的制备及其在缓/控释应用中的新进展
}

\author{
鲍艳, 王 䑣 \\ (陕西科技大学 轻工科学与工程学院, 西安 710021)
}

摘 要: 中空二氧化硅 $\left(\mathrm{SiO}_{2}\right)$ 微球具有特殊的内部空腔、吸附渗透性好、物质传递可控等优异性能，可储存负载并缓 慢释放药物、香精、染料、菌素等客体分子, 因此在药物缓释、医学成像、环境保护以及化妆品等领域有着广阔的 应用前景。根据国内外研究进展, 本文归纳对比了中空 $\mathrm{SiO}_{2}$ 微球几种制备方法之间的优劣差异, 着重阐述了其作 为缓控释载体表现出的持久性和高效性, 以及功能化的有机/无机杂化微球在响应性控释方面的优越性。并对中空 $\mathrm{SiO}_{2}$ 微球作为新型缓控释载体的发展前景进行了展望。

关 键 词: 中空 $\mathrm{SiO}_{2}$ 微球; 传统模板法; 自模板法; 缓释; 响应性控释; 综述

中图分类号: TB34 文献标识码: A

\section{Recent Advances in Fabrication and Sustained/Controlled-release Application of Hollow Silica Microspheres}

\author{
BAO Yan, WANG Tong
}

(College of Bioresources Chemistry and Materials Engineering, Shaanxi University of Science and Technology, Xi'an 710021, China)

\begin{abstract}
Hollow silica $\left(\mathrm{SiO}_{2}\right)$ microspheres with special interior spaces, excellent adsorption and permeability, and controllability of material deliveries are suitable for loading and releasing guest molecules of various functionalities such as drug, fragrances, dyes, bacteria, etc. More importantly, hollow silica $\left(\mathrm{SiO}_{2}\right)$ microspheres have attracted much attention due to their tremendous applications in sustained drug release, medical imaging, environmental protection, and cosmetics industry. In this paper, different preparation methods of hollow silica microspheres are reviewed and compared. Besides, the persistence and efficiency of hollow silica microspheres employed as delivery carriers and superiority of functionalized organic/inorganic hybrid microspheres are emphatically summarized. Meanwhile, the vast potential for future development of hollow silica microspheres as a novel type of sustained/controlled release carriers is prospected.
\end{abstract}

Key words: hollow silica microspheres; traditional template method; self-template method; sustained release; responsive controlled release; review

中空微球不仅具有结构规整、尺寸均一、比表 面积大(内外两个表面)、密度小、化学稳定性高等 特点, 而且具有特殊的中空结构, 使其具有独特的 光、电、热、磁、光电化学、机械以及催化性能 ${ }^{[1]}$, 因 而在生物医药、电子、能源和环保等领域有着广阔
的应用前景 ${ }^{[2-6]}$ 。一直以来, 中空 $\mathrm{SiO}_{2}$ 微球的制备和 应用都是科学家们研究的重点, 在学术界以及工业 领域掀起了极大的热潮。中空 $\mathrm{SiO}_{2}$ 微球不仅具有无 毒、价廉、资源丰富、高稳定、易改性等一系列优 点 ${ }^{[7-8]}$, 而且具有特殊的中空结构, 可储存负载并缓 
慢释放客体分子，产生 “包裹” 效应 ${ }^{[9]}$, 实现特殊材 料的可控传递。例如中空 $\mathrm{SiO}_{2}$ 微球可作为药物、基 因、蛋白质、香精、染料、菌素、脂类、酶、活细 胞等物质的存储和缓控释载体 ${ }^{[10-13]}$ 。此外, 随着科 技的日益进步, 纯的中空 $\mathrm{SiO}_{2}$ 微球已经不能满足应 用的需要, 因此功能化的复合材料应运而生。中空 $\mathrm{SiO}_{2}$ 微球表面含有硅羟基, 易于氨基、羧基或放基 等进行修饰，不仅可以更有效地控制药物扩散，而 且功能化的有机/无机杂化中空 $\mathrm{SiO}_{2}$ 微球还具有更 低的溶血性和更好的组织相容性 ${ }^{[14]}$, 因此可用于调 节折射率 ${ }^{[15]}$, 提高粒子承受体积周期性变化的能 力 $^{[16]}$, 以及扩大成像标记阵列以便于癌症的早期探 测 ${ }^{[17]}$ 等方面, 解决了人类健康和环境保护等重大问 题。本文着眼于国内外中空 $\mathrm{SiO}_{2}$ 微球制备的最新进 展, 重点综述了中空 $\mathrm{SiO}_{2}$ 微球在缓控释领域的独特 优越性。

\section{1 中空 $\mathrm{SiO}_{2}$ 微球的制备}

中空 $\mathrm{SiO}_{2}$ 微球的研究经历了较长的探索时期, 早在 1884 年, Kowalski 等 ${ }^{[18]}$ 就对其进行了大量的研 究。经过若干年的发展, 中空 $\mathrm{SiO}_{2}$ 微球的制备已经 有传统模板法 ${ }^{[19]}$ 、自模板法 ${ }^{[20] 、}$ 水热合成法 ${ }^{[21-22] ~}$ 和喷雾干燥法 ${ }^{[23-25]}$ 等。水热合成法是在高温高压下, 水及其他溶剂处于亚临界或超临界状态, 化学反 应的活性提高，材料间互相反应形成空心微球的

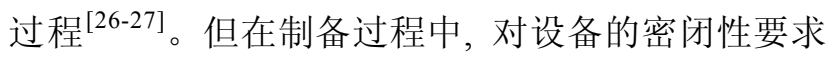
较高, 且反应温度和反应时间均对空心微球的粒径 和结构具有较大影响, 导致微球的均匀性较差。因 此将水热法和模板-界面反应法相结合, 既可以提 高模板核与反应物之间的反应活性, 又可以通过调 节反应温度、反应时间和模板核尺寸来控制空心微 球的形貌和粒径。该方法的缺点是水热处理过程中 产生向外扩张的驱动力较大, 压力促使模板和壳层 骨架协同向外扩张, 导致粒径增大。喷雾干燥法是 一种典型的制备中空微球的物理方法, 具有独特的 优势, 例如制备过程连续, 反应过程无污染, 产物 纯度较高, 适用于连续化大规模生产等, 但是所制 备的中空 $\mathrm{SiO}_{2}$ 微球粒径较大, 一般介于亚微米到十 微米之间, 且对制备工艺和设备的依赖性较强, 因 此在应用上也受到一定限制 ${ }^{[23]}$ 。传统模板法和自模 板法则是迄今为止应用最为广泛的两类方法。

\section{1 传统模板法}

传统模板法是以特定的物质作为模板, 利用前 驱体在一定介质和催化剂条件下进行水解缩合反应,
从而在模板表面形成相应无机物层，最后将模板去 除得到目标产物的方法, 如图 1 所示 ${ }^{[28]}$ 。在壳层的 生成过程中, 通常涉及到溶胶一凝胶(Sol-Gel)和层层 自组装(LBL)两类技术。溶胶一凝胶技术是利用有机 硅烷的水解缩合反应, 硅源在模板表面发生溶胶凝胶化, 得到中空 $\mathrm{SiO}_{2}$ 微球的过程 ${ }^{[29-30]}$, 具有所需 温度较低、反应容易进行、分散性好等优点，但原 材料价格较高、反应时间较长。层层自组装技术是 利用不同带电物质之间的静电吸附作用，在模板材 料上层层沉积相互之间有驱动力的物质的过程 ${ }^{[31]}$, 中空 $\mathrm{SiO}_{2}$ 微球的壳层厚度可以通过改变包覆周期 次数来调节, 而空心尺寸则通过调节模板尺寸得以 控制。该方法具有操作简单、设备要求低、制备环境 温和、选材广泛等优点, 但很难制备粒径小于 $200 \mathrm{~nm}$ 的中空 $\mathrm{SiO}_{2}$ 微球, 并且需在苛刻条件下才能去除模 板, 给实验设计和操作带来很大的不便 ${ }^{[32]}$ 。根据模 板物理形态的不同，传统模板法又分为以共价键维 持其特定结构的硬模板法和以分子间或分子内相互 作用维持其特定结构的软模板法两类 ${ }^{[33-34]}$ 。

\subsection{1 硬模板法}

硬模板法通常是以聚合物颗粒、无机非金属粒 子或者金属粒子为模板, 通过表面包裹先形成核壳 结构的复合微球, 然后采用酸碱溶解或高温敖烧的 方法去除模板，从而得到中空微球的过程 ${ }^{[35-36]}$ 。硬 模板法是中空微球制备过程中使用最早, 工艺最成 熟，应用范围最广的一种方法。

以聚合物颗粒为模板制备中空 $\mathrm{SiO}_{2}$ 微球是较为 常见的一种方法。Zou 等 ${ }^{[37]}$ 以聚苯乙烯(PS)微球为模 板, 采用聚乙烯吡咯烷酮(PVP)对 PS 微球进行改性, 在醇/水混合溶剂中, 以正硅酸乙酯(TEOS)为硅源, 水解生成 $\mathrm{SiO}_{2}$ 包覆在 $\mathrm{PS}$ 表面, 形成 $\mathrm{PS} / \mathrm{SiO}_{2}$ 核壳结 构, 在 $450^{\circ} \mathrm{C}$ 下煅烧 $1 \mathrm{~h}$ 去除模板, 获得结构规整的中 空 $\mathrm{SiO}_{2}$ 微球。在此基础上, $\mathrm{Bao}$ 等 ${ }^{[38]}$ 首先采用乳液聚 合法制备了 PS 微球, 然后以其为模板, TEOS/乙烯基 三乙氧基硅烷(VTES)为硅源，成功制备了结构规整、 浑圆度高的介孔中空 $\mathrm{SiO}_{2}$ 微球, 如图 2 所示。该微球 粒径分布较窄, 空腔直径约为 $150 \mathrm{~nm}$, 壳层厚度在 3 40 nm 之间, 且壳层具有大量介孔。

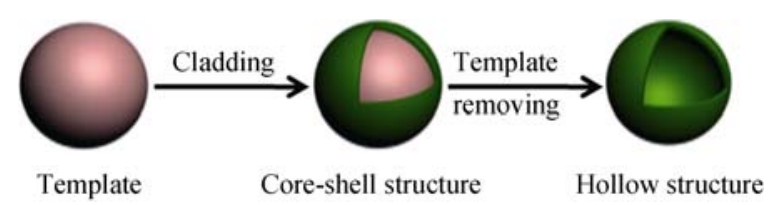

图 1 传统模板法制备中空 $\mathrm{SiO}_{2}$ 微球示意图 ${ }^{[28]}$

Fig. 1 A schematic of the process for hollow silica microspheres preparation via traditional templating method ${ }^{[28]}$ 


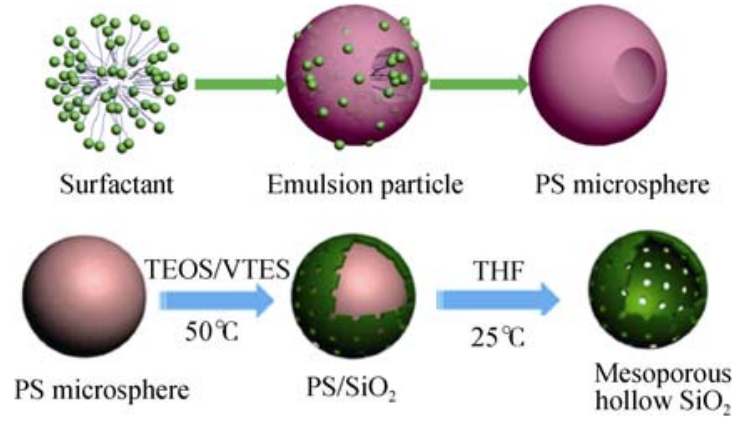

图 2 以 $\mathrm{PS}$ 微球为模板制备介孔中空 $\mathrm{SiO}_{2}$ 微球的示意图 ${ }^{[38]}$

Fig. 2 A schematic of the process for mesoporous hollow silica microspheres preparation via PS template ${ }^{[38]}$

由于中空 $\mathrm{SiO}_{2}$ 微球密度小, 具有内外两个表面, 因此相较于一般的 $\mathrm{SiO}_{2}$ 微球更加易于发生团聚。针 对这一现象, Suhendi 等 ${ }^{[39]}$ 采用一种全新的方法制备 无团聚的中空 $\mathrm{SiO}_{2}$ 微球, 其创新之处在于: 去除 PS 模板前先对 $\mathrm{SiO}_{2}$ 微球进行电喷射沉积。实验结果表 明, 通过改变模板 PS 球的尺寸、电喷雾的流量以及 前驱体的浓度可以控制中空 $\mathrm{SiO}_{2}$ 微球的聚集状况。

在无机非金属中, 碳(C)纳米球因其表面富含 羟基、粒径均一、价格低廉等常被用作制备中空微 球的模板。例如 Chen 等 $^{[40]}$ 以碳 $(\mathrm{C})$ 纳米球为模板, 采用 “三步法” 制备复合中空纳米微球。首先以葡 萄糖为原料合成碳纳米球, 然后在其表面均匀负载 直径为 $5 \mathrm{~nm}$ 的钯 $(\mathrm{Pd})$ 纳米球, 再以 TEOS 为硅源, $\mathrm{CTAB}$ 为孔模板, 得到 $\mathrm{SiO}_{2}$ 包覆的 $\mathrm{Pd} / \mathrm{C}$ 球, 最后通 过㷽烧去除 $\mathrm{C}$ 纳米球和 $\mathrm{CTAB}$ 等有机物, 制备得到 负载 $\mathrm{Pd}$ 纳米粒子的介孔中空 $\mathrm{SiO}_{2}$ 复合微球。

由上可以看出, $\mathrm{C}$ 纳米球作为模板材料虽然单 分散性好, 但其去除过程涉及到高温敾烧, 最终导 致中空 $\mathrm{SiO}_{2}$ 微球发生团聚、壳层坍塌等问题。因此, 寻找单分散性良好、粒径较小且易于去除的模板成 为研究热点。基于此, 鲍艳等 ${ }^{[41]}$ 首先通过聚乙烯吡 咯烷酮(PVP)和吐温-80 对粒径为 $30 \mathrm{~nm}$ 左右的氧化 锌 $(\mathrm{ZnO})$ 进行改性, 再以其为模板, 在超声及表面活 性剂的作用下, 通过 TEOS 原位水解制备核/壳型 $\mathrm{ZnO} / \mathrm{SiO}_{2}$ 复合微球, 最后在盐酸溶液中溶解 $\mathrm{ZnO}$ 核, 成功制备了粒径为 $47 \mathrm{~nm}$ 左右的中空 $\mathrm{SiO}_{2}$ 微球, 其 壳层厚度为 $12 \mathrm{~nm}$, 空腔直径为 $23 \mathrm{~nm}$, 有效解决了 上述难题。

\subsection{2 软模板法}

软模板法通常是以有机大分子、生物 ${ }^{[42]}$ 、表面 活性剂形成的液晶和胶束等为模板, 利用界面反应 和分子间作用力在界面进行规律性组装, 进而得到 中空微球。其中, 以表面活性剂形成的胶束和乳液 为模板最为常见, 其特点是用量很少即可大大降低
溶剂的表面张力，同时改变体系的界面组成和结构, 防止原生粒子团聚 ${ }^{[43]}$ 。

鲍艳等 ${ }^{[44]}$ 采用阳离子表面活性剂十六烷基三 甲基溴化铵(CTAB)形成的胶束为软模板, TEOS 和 $\gamma$-氨丙基三乙氧基硅烷(KH-550)为混合硅源, 氨水 为催化剂制备了表面亲水的中空 $\mathrm{SiO}_{2}$ 微球, 如图 3(a)所示。从图 3(b、c) 可以看出, 试验成功获得了 结构规整、粒径分布均匀、具有明显中空结构的 $\mathrm{SiO}_{2}$ 微球。

在以表面活性剂形成的胶束为模板的过程中, 其结构极大影响着胶束化的进程及其所形成的介孔 相。与普通单链表面活性剂相比, 双子表面活性剂 具有结构灵活多变、临界胶束浓度低、胶束聚集形 态多样等特点。鉴于此, 李敏 ${ }^{[45]}$ 以季铵盐型双子表 面活性剂为模板，通过简单控制双子表面活性剂在 反应溶液中的动力学自组装过程, 只使用一种表面 活性剂和单一硅源, 并在一种溶剂中成功制备粒径 均匀、直径约为 $250 \mathrm{~nm}$ 的规则球体。TEM照片进 一步表明这些球体都是中空结构的, 存在着由约 $50 \mathrm{~nm}$ 厚的固体 $\mathrm{SiO}_{2}$ 壳包围的均匀空腔。

以胶束为模板的软模板法不仅在制备具有完全 空腔结构的 $\mathrm{SiO}_{2}$ 微球上具有一定优势, 在制备含芯 中空 $\mathrm{SiO}_{2}$ 微球上也独树一帜。鉴于传统制备方法的 复杂性以及结构的特定性、单一性等缺陷, $\mathrm{Wu}$ 等 ${ }^{[46]}$ 选择合适的混合表面活性剂为模板，采用一步法制 备了如图 4 所示的多种类型的含芯中空 $\mathrm{SiO}_{2}$ 微球。 这种内部功能化的构思取代了在壳层表面接枝改性 的繁琐步骤, 并且壳层能够有效地预防芯粒子发生 聚集, 这在化学、光学和生物医学等领域具有极大 的应用前景。

1999 年, Jafelicci 等 ${ }^{[47]}$ 通过控制硅酸钠在庚烷AOT-水反相微乳液中的聚合反应，制备了微小的中

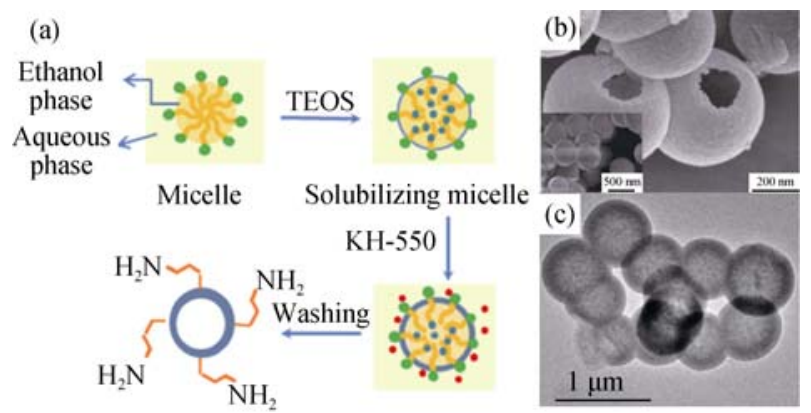

图 3 (a)软模板法制备中空 $\mathrm{SiO}_{2}$ 微球的机理图; 中空 $\mathrm{SiO}_{2}$ 微 球的(b)SEM 和(c)TEM 照片 ${ }^{[44]}$

Fig. 3 (a) A schematic of the process for hollow silica microspheres preparation via soft-templating method; (b) SEM and (c)TEM images of hollow silica microspheres ${ }^{[44]}$ 


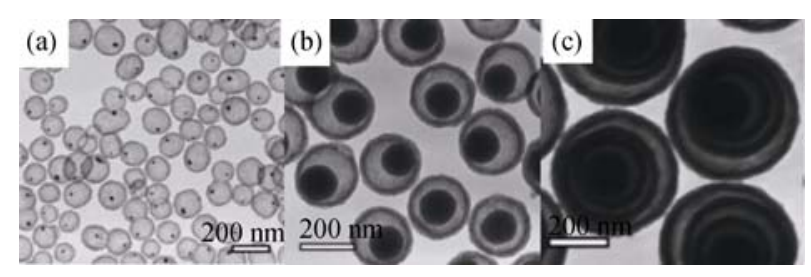

图 4 (a) 金 $/ \mathrm{SiO}_{2}$ 摇铃状中空微球, (b) $\mathrm{SiO}_{2} / \mathrm{SiO}_{2}$ 摇铃状中空微 球和(c) $\mathrm{SiO}_{2} / \mathrm{SiO}_{2}$ 多重壳层状中空微球的 TEM 照片 ${ }^{[46]}$

Fig. 4 TEM images of (a) $\mathrm{Au} / \mathrm{SiO}_{2}$ yolk/shell hollow silica microspheres, (b) $\mathrm{SiO}_{2} / \mathrm{SiO}_{2}$ yolk/shell hollow silica microspheres and (c) $\mathrm{SiO}_{2} / \mathrm{SiO}_{2}$ multilayer shell hollow silica microspheres $^{[46]}$

空 $\mathrm{SiO}_{2}$ 球形颗粒。从此, 乳液法制备中空微球逐渐 进入研究者们的视野。乳液是由两种互不相容的液 体混合均匀组成的稳定体系, 其中一相为分散相 (液滴), 另一相为连续相(分散介质), 常见的是油分 散于水中 $(\mathrm{O} / \mathrm{W})$ 或水分散在油中 $(\mathrm{W} / \mathrm{O})$ 。乳液法以乳 液液滴为模板, 利用硅烷与不同溶剂之间的极性差 异在两相界面发生水解、缩合等化学反应, 形成稳 定的胶体粒子包覆在乳液液滴表面, 进而得到中空 结构 $\mathrm{SiO}_{2}$ 微球 ${ }^{[48]}$ 。Teng 等 ${ }^{[49]}$ 以 CTAB表面活性剂稳 定和诱导TEOS油滴在醇水溶液的水解作用, 通过 溶胶一凝胶反应制备了介孔中空 $\mathrm{SiO}_{2}$ 微球。通过改变 醇水比, 合成了粒径范围为 210 720 $\mathrm{nm}$ 的中空球, 并通过改变体系中 CTAB的浓度来调节壳层的厚度, 所制备的介孔中空 $\mathrm{SiO}_{2}$ 微球的比表面积范围为 924 $1766 \mathrm{~m}^{2} / \mathrm{g}$ 。此外, 近年来双重乳液因其独特的三相结 构已成为制备中空 $\mathrm{SiO}_{2}$ 微球的新型模板 ${ }^{[50]}$ 。Chul等 ${ }^{[51]}$ 采用 $\mathrm{O} / \mathrm{W} / \mathrm{O}$ 型双重乳液为模板, 在外油相中加入 TEOS, 通过TEOS的扩散, 使其在中间水相中发生 水解形成 $\mathrm{SiO}_{2}$, 最终制得中空 $\mathrm{SiO}_{2}$ 微球。

与其它模板相比, 乳液模板去除过程较为简 单，但反应时间必须严格控制才能得到中空结构 的微球, 且对于特定的 “油” 相和 “水” 相, 达到 稳定状态时所能得到的微球尺寸和壁厚难以调节, 摚拌速度和乳液 $\mathrm{pH}$ 的要求都较为苛刻, 因此有待 进一步改进。

综上可知, 采用传统模板法制备中空微球的过 程中模板的去除步骤是不可缺少的, 而模板的去除 又是非常复杂的过程, 其对所生成的中空微球的形 貌和结构等均有不利影响 ${ }^{[52]}$ 。因此, 探寻更加简单 有效的中空微球的合成方法是非常必要的。

\section{2 自模板法}

自模板法是一类先合成微/纳米尺度的 “模板”, 再将其转变为介孔空心结构的方法。与传统模板法 的区别在于：其 “模板” 不仅起到传统模板的支撑
框架作用，还直接参与到壳层的形成过程中，“模板” 材料直接转化为壳层或者作为壳层的前驱物 ${ }^{[53-55]}$ 。

Zhang 等 ${ }^{[56]}$ 选用 $\mathrm{NaOH}$ 作为刻蚀剂, PVP 作为 保护剂, 采用表面保护刻蚀的方法成功将实心 $\mathrm{SiO}_{2}$ 微球转化为介孔中空 $\mathrm{SiO}_{2}$ 微球。刻蚀过程中, 初始 的单分散实心球表面被 PVP 保护, $\mathrm{OH}^{-}$扩散至 $\mathrm{SiO}_{2}$ 微球内部, 使其内部具有相对较高的刻蚀速率, 连 续刻蚀下最终产生介孔中空结构。

$\mathrm{Hu}$ 等 ${ }^{[57]}$ 通过 TEOS 与硅烷偶联剂的共水解缩 合作用，采用简单的自模板法成功制备了中空 $\mathrm{SiO}_{2}$ 微球。如图 5 所示, 在氨水的催化下, 乙烯基三甲氧 基硅烷(VTMS)偶联剂首先预水解形成胶束, 随着 TEOS 的加入, $\mathrm{Si}-\mathrm{OH} 、 \mathrm{CH}_{3} \mathrm{O}-$ 与 TEOS 发生溶胶凝胶反应，少部分 $\mathrm{SiO}_{2}$ 微粒溶解在胶束内部，大部 分则在胶束表面形成多孔 $\mathrm{SiO}_{2}$ 壳层, 无需模板去除 过程即可合成乙烯基中空 $\mathrm{SiO}_{2}$ 微球。其创新之处在 于硅烷偶联剂不仅作为模板, 也作为 $\mathrm{SiO}_{2}$ 的表面改 性剂, 既简化了制备过程, 又赋予了中空 $\mathrm{SiO}_{2}$ 微球 更多的功能，使其具有优异的吸附性能。

自模板法的优势还在于它能简易地制备具有独 特结构的中空 $\mathrm{SiO}_{2}$ 微球，“雕刻” 不同种类和功能 的微纳结构材料, 获得让人兴奋不已的雕刻艺术 品。例如 Tang 等 ${ }^{[58]}$ 通过 TEOS 与氮[3-(三甲氧基硅 基)丙基]乙二胺(TSD)的共缩合作用，首先获得三明 治结构的 $\mathrm{SiO}_{2}-\mathrm{TSD} / \mathrm{SiO}_{2}$-实心 $\mathrm{SiO}_{2}$ 微球和核壳结构 的 $\mathrm{TSD} / \mathrm{SiO}_{2}$-实心 $\mathrm{SiO}_{2}$ 微球, 再分别以其为模板, 通过选择性刻蚀合成了摇铃结构介孔中空 $\mathrm{SiO}_{2}$ 微 球以及介孔中空 $\mathrm{SiO}_{2}$ 微球, 如图 6 所示。

为了精确地控制中空介孔 $\mathrm{SiO}_{2}$ 微球的组成并 赋予其更多功能，有机/无机杂化中空 $\mathrm{SiO}_{2}$ 微球的 制备广受关注 ${ }^{[59]}$ 。Chen 等 ${ }^{[60]}$ 采用硅刻蚀化学法制 备了壁层组成可调控的有机/无机杂化中空 $\mathrm{SiO}_{2}$ 微

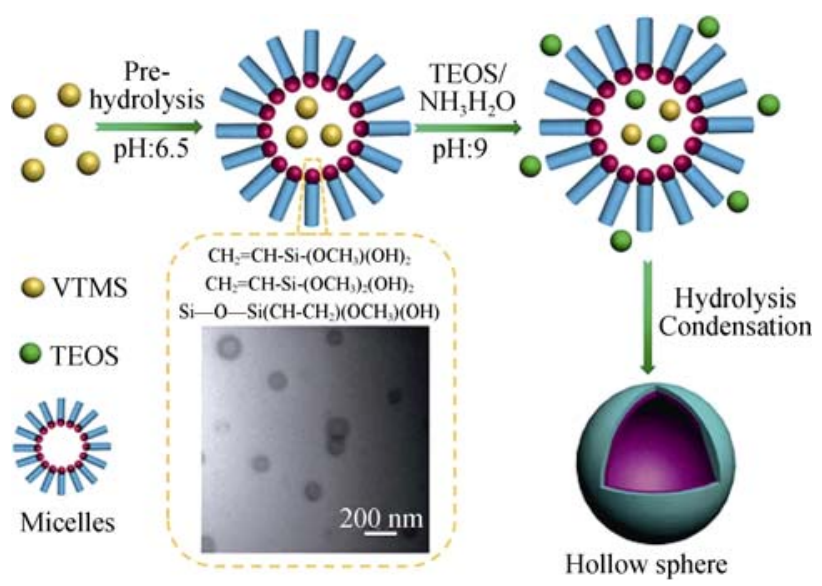

图 5 乙烯基中空 $\mathrm{SiO}_{2}$ 微球的形成机理示意图 ${ }^{[57]}$

Fig. 5 Formation mechanism of vinyl silica hollow spheres ${ }^{[57]}$ 


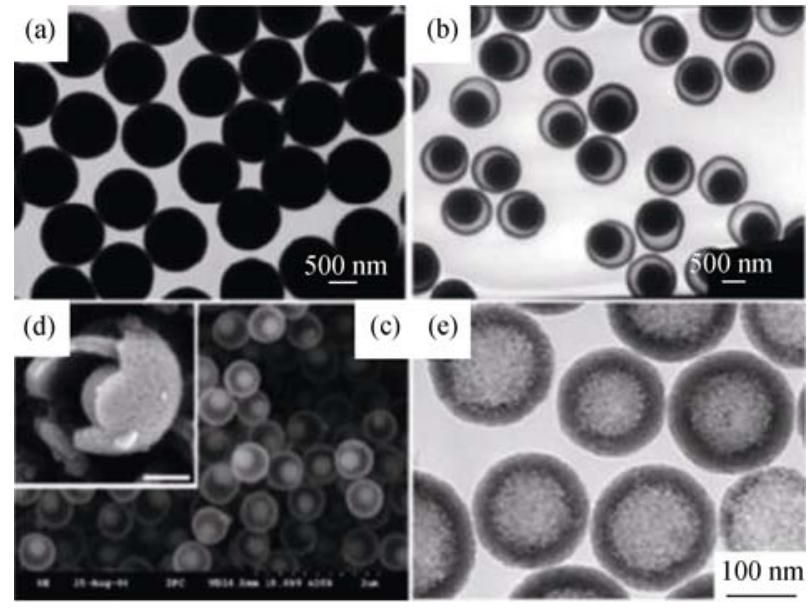

图 6 (a) 实心 $\mathrm{SiO}_{2}$ 微球的 TEM 照片, 摇铃结构介孔中空 $\mathrm{SiO}_{2}$ 微球的(b)TEM 照片和(c、d)SEM 照片和(e)介孔中空 $\mathrm{SiO}_{2}$ 微 球的 TEM 照片 ${ }^{[58]}$

Fig. 6 (a) TEM image of solid silica spheres, (b) TEM and (c, d) SEM images of mesoporous hollow silica nanorattles, (e) TEM image of mesoporous hollow silica microspheres ${ }^{[58]}$

球。其独到之处在于: 利用有序介孔中空有机硅纳 米粒子的 “拓扑变换” , 通过 “原位框架热解法” 将有机硅壳表面的亚苯基转变为碳骨架, 从而获得 均一的 $\mathrm{SiO}_{2} / \mathrm{C}$ 杂化中空微球。杂化中空微球具有可 调控的生物降解性、良好的生物相容性, 并可递送 疏水性药物从而抑制癌细胞的转移, 在纳米加工、 纳米生物技术以及催化方面具有重要应用。

此外, 形貌特异的中空微球也是一大亮点, Ji 等 ${ }^{[61]}$ 采用自模板法制备了如图 7 所示的火花壳状中 空 $\mathrm{SiO}_{2}$ 微胶囊, 其在适当的 $\mathrm{pH}$ 条件下壳层可灵活 转变, 具有可调的结构和性能。Terentyeva 等 ${ }^{[62]}$ 将 采用上述方法制备的火花壳状中空 $\mathrm{SiO}_{2}$ 微胶囊用 于固定化酶的应用。

综上可知，虽然传统模板法和自模板法是制备

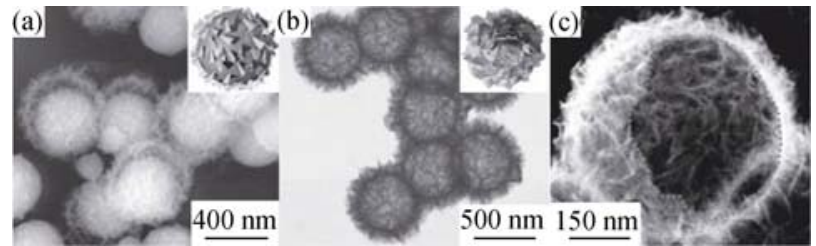

图 7 火花壳状 $\mathrm{SiO}_{2}$ 微胶囊的 $\mathrm{SEM}$ 照片 ${ }^{[61]}$

Fig. 7 SEM images of hollow silica microcapsule ${ }^{[61]}$

中空 $\mathrm{SiO}_{2}$ 微球过程中最常采用的两类方法, 但是其 也各有优缺点，具体总结见表 1 所示。

\section{2 中空 $\mathrm{SiO}_{2}$ 微球在缓控释中的应用}

\section{1 中空 $\mathrm{SiO}_{2}$ 微球在缓释方面的应用}

缓释是一个通过扩散作用实现缓慢持续释放的 过程，在日常生活中也常常见到，如缓释片、缓释胶 囊等, 可起到长久且高效的作用, 减少资源浪费。中 空 $\mathrm{SiO}_{2}$ 微球作为一种内部具有特殊中空结构的新 型材料, 具有均匀且可调控的孔径, 常见孔径尺寸 分布在 2 15 $\mathrm{nm}$ 之间, 而通常封装的客体分子的大 小为一个或几个纳米, 因此能够在介孔孔道中自由 进出, 实现客体分子的担载和释放, 并可通过改变 其结构来调节客体材料的装载量及释放速度 ${ }^{[63-66]}$, 是一种良好的新型缓释载体。中空 $\mathrm{SiO}_{2}$ 微球壁层中 如果没有良好的孔道结构和可控的孔径大小, 将会 极大地限制其在缓控释中的应用。有研究表明由于 空间位阻效应的影响, 孔径的减小会导致载药量和 释放速率的降低, 例如, 将介孔 $\mathrm{SiO}_{2}$ 的孔径从 $8.2 \mathrm{~nm}$ 增大到 $11.4 \mathrm{~nm}$, 牛血清蛋白的担载量将会从 $15 \%$ 上升到 $27 \%{ }^{[67]}$ 。而孔道结构指的是孔的连通性和几 何结构。对于扩散过程来说, 相互连通的孔道结构 比非连通的孔道结构更有利于缓释的进行。

表 1 各种制备方法的优劣对比

Table 1 Characteristics and limitations of various preparation methods

\begin{tabular}{cll}
\hline Method & \multicolumn{1}{c}{ Characteristic } & \multicolumn{1}{c}{ Limitation } \\
\hline \multirow{3}{*}{$\begin{array}{c}\text { Hard } \\
\text { template }\end{array}$} & (1) Adjustable particle size; & (1) Difficult to remove the templates \\
& (3) Uniform product morphology; & (2) The shell easy to collapse; \\
& (4) Foreseeable and high repetition rate & (3) Time-consuming and money-consuming \\
Soft & (1) Easy to prepare and remove the templates & (1) Poor structural stability and monodispersity; \\
template & (2) Simple technology; & (2) Not easy to control shell thickness; \\
& (3) Time-saving & (3) Wide particle size distribution; \\
Self & (1) No additional template; & (4) Low efficiency \\
template & (2) Simple step and time-saving synthetic; & (1) Various and complex influence factors; \\
& (3) High repetition rate; & (2) Large dependability on environment
\end{tabular}


Chen 等 ${ }^{[68]}$ 用中空 $\mathrm{SiO}_{2}$ 微球负载治癌药物并进 行靶向给药。由于治癌药物 5-氟尿嘧啶(5-FU)的 尺寸只有 $0.5 \mathrm{~nm}$, 而所制备的中空 $\mathrm{SiO}_{2}$ 微球的孔 径约为 $2.5 \mathrm{~nm}$, 因此, 通过物理吸附法将药物成功 负载到中空 $\mathrm{SiO}_{2}$ 微球的内部, 且其负载率高达 $194.5 \mathrm{mg}_{(5-\mathrm{FU})} / \mathrm{g}_{(\mathrm{HMSN})}$ 。在药物释放实验中发现, $0 \sim 1 \mathrm{~h}$ 时药物释放速度较快, 这是由于吸附在表面和介孔 孔道处的 5-FU 优先进行扩散; $1 \mathrm{~h}$ 后, 释放速度减慢; 约 $25 \mathrm{~h}$ 释放曲线趋于平稳。此外, 在浓度为 $5 \mu \mathrm{g} / \mathrm{mL}$ 时, 纯 5-FU 和具有缓释功能的 $5-\mathrm{FU} / \mathrm{SiO}_{2}$ 对大肠癌 细胞的生长抑制分别为 $42 \%$ 和 $28 \%$; 随着浓度的增加, 两者的细胞活性均有所降低, 当浓度达到 $50 \mu \mathrm{g} / \mathrm{mL}$, 两者对癌细胞的生长抑制作用均为 $58 \%$ 。由此可见， 载体递送药物是一个缓慢的过程, 并不会掩盖药物 本身的功效。

以上封装客体材料的方法多为 “浸渍法” [69-71], 即首先制备出中空 $\mathrm{SiO}_{2}$ 微球, 再将其浸入到客体材 料或其分散液中, 通过物理吸附作用来负载客体材 料。但由于中空 $\mathrm{SiO}_{2}$ 微球的孔径大小决定了可吸附 到孔内的客体分子的尺寸大小, 因此只有少数小尺 寸的分子可通过壳层上的介孔渗透到中空 $\mathrm{SiO}_{2}$ 微球 的内部, 比中空 $\mathrm{SiO}_{2}$ 微球孔径大的大尺寸分子则难 以被引入到空腔中 ${ }^{[72]}$ 。所以浸渍法封装效率较低, 客体材料前期释放速度过快, 持续释放效果相对较 差, 而且对客体材料的亲水性要求较高。为了解决 上述缺陷, Wang等 ${ }^{[73]}$ 采用直接封装法同时实现了载 体中空 $\mathrm{SiO}_{2}$ 微球的形成和药物分子在微球内的封 装。将水溶性药物溶解在 $\mathrm{W} / \mathrm{O}$ 型乳液的水相中, 用 含有药物的乳液滴作为模板, 利用硅源在 $\mathrm{W} / \mathrm{O}$ 乳液 界面上发生水解缩合反应来直接封装药物分子。结 果表明只有少量药物分子未进入微球内部而被封装 在硅壳的孔里, 大多数的药物分子则进入到中空 $\mathrm{SiO}_{2}$ 微球的空心部分。缓释曲线显示, 初始 $2 \mathrm{~h}$ 内药 物快速释放, 释放量达到近 $58 \%$, 这有利于药物浓 度快速上升到有效的水平。在接下来长时间的释放 周期内，释放速率大大下降, $60 \mathrm{~h}$ 之内从 $58 \%$ 缓慢增 加到近 $75 \%$, 这样可以维持药物浓度以满足长期治 疗效果的要求。直接封装法可以根据客体材料的亲 疏水性来选择模板的类型, 简化药物递送系统的工 艺, 对药物的装载效率远高于浸渍法, 且具有更优 异的缓释特性。

\section{2 中空 $\mathrm{SiO}_{2}$ 微球在控释方面的应用}

新型药物释放系统已经成为药学领域的重要发 展方向。为了避免普通释药系统存在的问题, 人们 提出了刺激响应给药系统的理念, 即利用载药体系
对释放环境或外部刺激的物理或化学响应情况控制 给药系统。这种刺激响应系统更智能化, 可以维持 药物在人体内的浓度, 并在特定的器官部位进行释 放 ${ }^{[74-75]}$ 。而为了实现客体分子的控制释放，则需要 在载体材料上修饰一些刺激响应的“开关分子”[76-77], 目前常见的刺激条件包括 $\mathrm{pH}^{[78]}$ 、温度 ${ }^{[79]}$ 、光 ${ }^{[80]}$ 、 生物分子 ${ }^{[81]}$ 、酶 ${ }^{[82]}$ 和DNA分子 ${ }^{[83]}$ 等。

桂万元等 ${ }^{[84]}$ 采用介孔中空 $\mathrm{SiO}_{2}$ 微球负载苂光染 料分子罗丹明 $\mathrm{B}(\mathrm{RhB})$, 并在其表面修饰溶菌酶分子 作为开关分子，构筑 $\mathrm{pH}$ 响应的药物控制释放系统, 通过观察在 $575 \mathrm{~nm}(\mathrm{RhB})$ 处荧光发射峰强度的变化 来估算其控制释放效果。如图8所示, $\mathrm{pH}$ 显著影响了 药物释放量。这是由于溶菌酶分子的有效尺寸随着 磷酸缓冲溶液 $\mathrm{pH}$ 的变化而变化, $\mathrm{pH}$ 升高, 水合半径 减小，“开关”打开，染料分子得以释放，反之亦然。 与普通介孔 $\mathrm{SiO}_{2}$ 微球相比, 中空结构的介孔 $\mathrm{SiO}_{2}$ 微 球负载量更大，释放量也明显增多，这说明其具有 良好的 $\mathrm{pH}$ 响应性控释能力。

环境响应性控释体系最常见的莫过于热响应。 $\mathrm{Hu}$ 等 ${ }^{[85}$ 采用 “一步法” 成功制备了热响应性聚氮 异丙基丙烯酰胺-苯乙烯(P(NIPAM-S))/ $\mathrm{SiO}_{2}$ 杂化中 空微球，并通过浸渍获得了负载香精的杂化中空 微球。因为PNIPAM在最低临界溶解温度(LCST)可 发生亲疏水转变, 当温度低于 $\operatorname{LCST}\left(28^{\circ} \mathrm{C}\right)$ 时, 聚 合物链传播和扩散到 $\mathrm{SiO}_{2}$ 壳外, 关闭孔隙并增加 球体的平均尺寸; 当温度高于 $28^{\circ} \mathrm{C}$ 时, 由共聚物链 组成的杂化中空球的核坞塌并且小孔开放，从而 实现了香精在特定环境中的热响应缓慢释放, 如 图9所示。在此研究基础上, $\mathrm{Hu}$ 等 $^{[86]}$ 还用上述杂化 中空微球负载维他命 $\mathrm{C}$, 也具有良好的温度控制释 放特性。

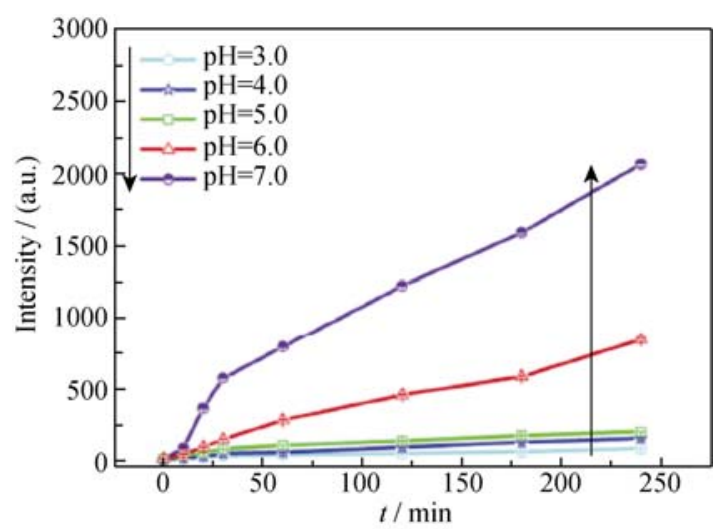

图 8 不同 $\mathrm{pH}$ 下介孔中空 $\mathrm{SiO}_{2}$ 微球的控制释放曲线图 ${ }^{[84]}$ Fig. 8 Controlled release curve under different $\mathrm{pH}$ of hollow mesoporous silica microspheres ${ }^{[84]}$ 


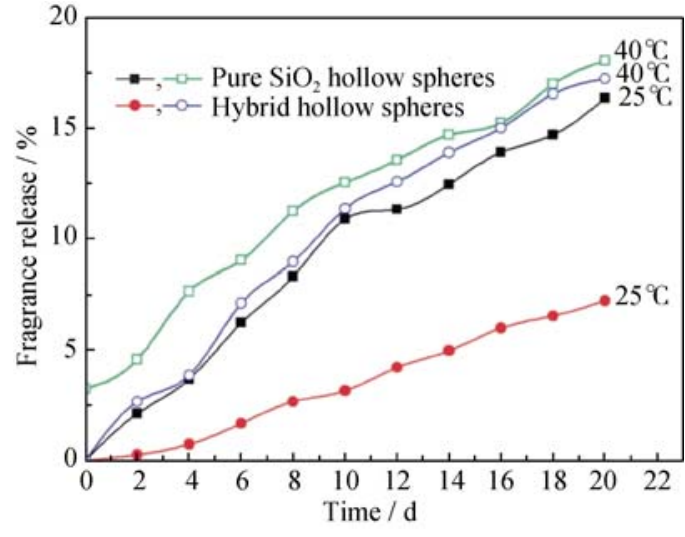

图 9 杂化中空微球和纯中空微球的香精释放曲线图 ${ }^{[85]}$

Fig. 9 Fragrance release of hybrid hollow spheres and the pure $\mathrm{SiO}_{2}$ hollow spheres ${ }^{[85]}$

$\mathrm{Zhu}$ 等 $^{[87]}$ 设计了一种新颖的酶诱导控制释放药 物和基因的方法。以荧光剂作为模型药物, 以胞嘧 啶核苷酸磷酸二酯酸 $(\mathrm{CpG} \mathrm{ODN})$ 作为模型基因, 以 聚( L - 赖氨酸)(PLL)作为酶诱导的可降解物, 其制备 思路为: 首先制备介孔中空 $\mathrm{SiO}_{2}$ 微球, 通过浸渍摚 拌负载菼光剂, 再用3-氨丙基三乙氧基硅烷对其表 面改性，制备了表面改性的负载苂光剂的介孔中空 $\mathrm{SiO}_{2}$ 微球; 然后将带负电的 $\mathrm{CpG}$ ODN和带正电的 PLL通过层层自组装包裹在介孔中空 $\mathrm{SiO}_{2}$ 微球外层, 制备PLL包裹的负载荧光剂和基因介孔中空 $\mathrm{SiO}_{2}$ 微 球; 最后, 在 $\alpha$-胰凝乳蛋白酶溶液中PLL降解, 苂 光剂和 $\mathrm{CpG}$ ODN得以释放。结果表明, 中空 $\mathrm{SiO}_{2}$ 微 球具有较高的药物负载能力和缓释效果, 其新颖之 处在于：以酶诱导的方式同时对药物和基因进行控 制释放，可通过改变溶液中酶的浓度来控制物质的 释放速率和累计释放量。这将在生物医药和癌症治 疗上具有非常可观的应用前景。

\section{3 总结与展望}

综上所述, 可以发现, 具有各项优异性能的中 空 $\mathrm{SiO}_{2}$ 微球在材料研究领域备受关注。其制备方法 多样化，无论是软、硬模板法还是自模板法，均具有 无可比拟的优势。在缓控释领域，中空 $\mathrm{SiO}_{2}$ 微球作 为一种新型载体材料, 对药物、香精、菌素、染料、 基因、蛋白质、酶以及生物分子等材料具有优良的 封装和缓释性能，而且近年来刺激响应性控释体 系的研究和探索更加突出了其潜在的应用价值。但 是其封装方法、封装率以及封装效率还有待进一步 改善。

目前, 中空 $\mathrm{SiO}_{2}$ 微球的研究仍局限在实验室阶
段，还有很多难题亟待解决，距离大规模生产尚有 差距。为了更好地实现产业化，笔者认为中空 $\mathrm{SiO}_{2}$ 微球的研究将趋于以下三个方向：(1)综合不同制备 方法的特点, 取其精华, 去其糟粕, 探索更加简单 的制备工艺，缩短制备时间; (2)实现中空 $\mathrm{SiO}_{2}$ 微球 形貌和尺寸的精确调控; (3)实现中空 $\mathrm{SiO}_{2}$ 微球的多 功能化改性，拓展其在更多领域的应用，将来中空 $\mathrm{SiO}_{2}$ 微球不仅可以封装上述所提及的物质, 还可以 封装任何小分子物质，甚至同时封装多种物质，并 实现在特定环境下的选择性缓慢释放。当代研究者 们应当秉承 “推陈出新，精益求精”的精神，以创新 驱动产业发展，让更多的新型材料快速融入市场新 环境，带来更大的效益。

\section{参考文献:}

[1] LIU J, LIU F, GAO K, et al. Recent developments in the chemica synthesis of inorganic porous capsules. Journal of Materials Chemistry, 2009, 19(34): 6073-6084.

[2] ZHANG C, HOU T, CHEN J, et al. Preparation of mesoporous silica microspheres with multi-hollow cores and their application in sustained drug release. Particuology, 2010, 8(5): 447-452.

[3] ZHU Y, SHI J, CHEN H, et al. A facile method to synthesize novel hollow mesoporous silica spheres and advanced storage property. Microporous and Mesoporous Materials, 2005, 84(1): 218-222.

[4] YANG X L, YAO K, ZHU Y H. Fabrication and sustained release property of nanostruetured hollow silica microspheres. Journal of Inorganic Materials, 2005, 20(6): 1403-1408.

[5] 鲍 艳, 杨永强, 马建中, 等. 一种聚丙烯酸酯/ 中空二氧化硅 纳米复合皮革涂饰剂的制备方法：中国，102704273A. 2012-10-03.

[6] ZHAO Y, SUN Y L, WANG H, et al. The application of hollow microspheres. Science and Technology in Chemical Industry, 2014, 22(5): $68-72$

[7] CHEN J F, DING H M, WANG J X, et al. Preparation and characterization of porous hollow silica nanoparticles for drug delivery application. Biomaterials, 2004, 25(4): 723-727.

[8] FENG X F, JIN W G, LIU F, et al. Advance in preparation of hollow mesoporous silica-based microsphere. Inorganic Chemical Industry, 2008, 40(12): 12-14.

[9] WANG J X, WANG Z H, CHEN J F, et al. Direct encapsulation of water-soluble drug into silica microcapsules for sustained release applications. Materials Research Bulletin, 2008, 43(12): 3374-3381

[10] SLOWING I, VIVERO E J, WU C, et al. Mesoporous silica nanoparticles as controlled release drug delivery and gene transfection carriers. Advanced Drug Delivery Reviews, 2008, 60(11): 
$1278-1288$.

[11] ZHU Y, SHI J, SHEN W, et al. Stimuli-responsive controlled drug release from a hollow mesoporous silica sphere/polyelectrolyte multilayer core-shell structure. Angewandte Chemie, 2005, 117(32): 5213-5217.

[12] LEE C, CHENG S, HUANG I, et al. Intracellular pH-responsive mesoporous silica nanoparticles for the controlled release of anticancer chemotherapeutics. Angewandte Chemie International Edition, 2010, 122(44): 8390-8395.

[13] CHANG F P, HUNG Y, CHANG J H, et al. Enzyme encapsulated hollow silica nanospheres for intracellular biocatalysis. ACS applied Materials \& Interfaces, 2014, 6(9): 6883-6890.

[14] CHEN Y, MENG Q, WU M, et al. Hollow mesoporous organosilica nanoparticles: a generic intelligent framework- hybridization approach for biomedicine. Journal of the American Chemical Society, 2014, 136(46): 16326-16334.

[15] RETSCH M, SCHMELAEISEN M, BUTT H J, et al. Visible Mie scattering in nonabsorbing hollow sphere powders. Nano Letters, 2011, 11(3): 1389-1394.

[16] JIN L, XU L, MOREIN C, et al. Titanium containing $\gamma-\mathrm{MnO}_{2}$ (TM) hollow spheres: one-step synthesis and catalytic activities in Li/Air batteries and oxidative chemical reactions. Advanced Functional Materials, 2010, 20(19): 3373-3382.

[17] ZHU Y, FANG Y, KASKEL S. Folate-conjugated $\mathrm{Fe}_{3} \mathrm{O}_{4} @ \mathrm{SiO}_{2}$ hollow mesoporous spheres for targeted anticancer drug delivery. The Journal of Physical Chemistry C, 2010, 114(39): 16382-16388.

[18] KOWALSKI A, VOGEL M, BLANKENSHIP R M. US Patent4, $427,836,1884$.

[19] ZHANG S, XU L, LIU H, et al. A dual template method for synthesizing hollow silica spheres with mesoporous shells. Materials Letters, 2009, 63(2): 258-259.

[20] WANG Y, TANG C, DENG Q, et al. A versatile method for controlled synthesis of porous hollow spheres. Langmuir, 2010, 26(18): 14830-14834.

[21] KATO N, KATO N. High-yield hydrothermal synthesis of mesoporous silica hollow capsules. Microporous and Mesoporous Materials, 2016, 219: 230-239.

[22] DUAN Y W, YU A H, ZHAI G X. Progress of preparation methods of hollow microspheres. Chinese Journal of Pharmaceuticals, 2015, 46(6): 639-646.

[23] SCHMID A, FUJII S, ARMES S P. Polystyrene silica nanocomposite particles via alcoholic dispersion polymerization using a cationic azo initiator. Langmuir, 2006, 22(11): 4923-4927.

[24] SEN D, KHAN A, BAHADUR J, et al. Use of small-angel neutron scattering to investigate modifications of internal structure in self-assembled grains of nanoparticles synthesized by spray drying. Journal of Colloid and Interface Science, 2010, 347(1): 25-30.
[25] HAGURA N, NANDIYANTO A B D, ISKANDAR F, et al. A role of template surface charge in the preparation of porous and hollow particles using spray-drying. Chemistry Letters, 2009, 38(11): 1076-1077.

[26] $\mathrm{HU} \mathrm{W}, \mathrm{DU} \mathrm{X}$, WU Y, et al. Novel $-\mathrm{Cu}_{0.95} \mathrm{~V}_{2} \mathrm{O}_{5}$ hollow microspheres and $a-\mathrm{CuV}_{2} \mathrm{O}_{6}$ nanograins: Facile synthesis and application in lithium-ion batteries. Journal of Power Sources, 2013, 237(237): 112-118.

[27] WEI J, DU A, JIN F, et al. The preparation and high-frequency electromagnetic properties of ferrimagnetic bisphthalonitrile- $\mathrm{Fe}_{3} \mathrm{O}_{4}$ core-shell hollow microspheres. Journal of Magnetism and Magnetic Materials, 2013, 340(8): 70-75.

[28] 杨永强. 聚丙烯酸酯/中空二氧化硅纳米复合皮革涂饰剂的制 备及应用研究. 陕西科技大学, 2014.

[29] GU W J, LIAO Y, WU W B, et al. Progress in the preparation of hollow silica spheres. Silicone Material, 2009, 23(4): 257-264.

[30] TENG Z, HAN Y, LI J, et al. Preparation of hollow mesoporous silica spheres by a Sol-Gel/emulsion approach. Microporous and Mesoporous Materials, 2010, 127(1): 67-72.

[31] BECKER AL, JOHNSTON APR, CARUSO F. Layer-by-layerassembled capsules and films for therapeutic delivery. Small, 2010, 6(17): $1836-1852$.

[32] XIE F, QI M Z, LI W J, et al. Classification, fabrication methods and applications of inorganic hollow spheres. Progress in Chemistry, 2011, 23(12): 2522-2533.

[33] MALGRAS V, JI Q, KAMACHI Y, et al. Templated synthesis for nanoarchitectured porous materials. Bulletin of the Chemical Society of Japan, 2015, 88(9): 1171-1200.

[34] HSUEH H Y, YAO C T, HO R M. Well-ordered nanohybrids and nanoporous materials from gyroid block copolymer templates. Chemical Society Reviews, 2015, 44(7): 1974-2018.

[35] NANDIYANTO A B D, KIM S G, ISKANDAR F, et al. Synthesis of spherical mesoporous silica nanoparticles with nanometer-size controllable pores and outer diameters. Microporous and Mesoporous Materials, 2009, 120(3): 447-453.

[36] YANG Z, CONG H, CAO W. Narrowly dispersed micrometersized composite spheres based on diazonium-polystyrene. Journal of Polymer Science Part A: Polymer Chemistry, 2004, 42(17): 4284-4288.

[37] ZOU H, WU S, RAN Q, et al. A simple and low-cost method for the preparation of monodisperse hollow silica spheres. The Journal of Physical Chemistry C, 2008, 112(31): 11623-11629.

[38] BAO Y, YANG Y Q, MA J Z. Fabrication of monodisperse hollow silica spheres and effect on water vapor permeability of polyacrylate membrane. Journal of Colloid and Interface Science, 2013, 407(10): $155-163$.

[39] SUHENDI A, NANDIYANTO A B, MUNIR M M, et al. Prepara- 
tion of agglomeration-free spherical hollow silica particles using an electrospray method with colloidal templating. Materials Letters, 2013, 106(9): 432-435.

[40] CHEN Z, CUI Z M, NIU F, et al. Pd nanoparticles in silica hollow spheres with mesoporous walls: a nanoreactor with extremely high activity. Chem. Commun., 2010, 46(35): 6524-6526.

[41] BAO Y, YANG Y Q, MA J Z, et al. Fabrication of hollow silica microsphere using zinc oxide as template and its effect on polyacrylate film properies. Journal of the Chinese Ceramic Society, 2014, 42(7): 914-925.

[42] CAO F, LI D X, GUAN Z S. Preparation of silica hollow microspheres with special surface morphology by biotemplate method. Journal of Inorganic Materials, 2009, 24(3): 501-506.

[43] WANG X, MIAO X R, LI Z M, et al. Fabrication of microporous hollow silica spheres templated by NP-10 micelles without calcinations. Applied Surface Science, 2011, 257(7): 2481-2488.

[44] BAO Y, SHI C H, MA J Z. Fabrication of hollow silica spheres and their effect on water vapor permeability of waterborne polyurethane film. Journal of the Chinese Ceramic Society, 2015, 43(1): $35-41$.

[45] 李 敏. 双子表面活性剂为模板制备中空二氧化硅纳米球及其 载药性能研究. 湖北: 华中科技大学博士学位, 2013.

[46] WU X J, XU D. Soft template synthesis of yolk/silica shell particles. Advanced Materials, 2010, 22(13): 1516-1520.

[47] JAFELICCI JR M, DAVOLOS M R, DOS SANTOS F J, et al. Hollow silica particles from microemulsion. Journal of Noncrystalline Solids, 1999, 247(1): 98-102.

[48] SINGH R K, GARG A, BANDYOPADHYAYA R, et al. Density fractionated hollow silica microspheres with high-yield by non-polymeric Sol-Gel/ emulsion route. Colloids and Surfaces A: Physicochemical and Engineering Aspects, 2007, 310(1): 39-45.

[49] TENG Z, HAN Y, LI J, et al. Preparation of hollow mesoporous silica spheres by a Sol-Gel/ emulsion approach. Microporous and Mesoporous Materials, 2010, 127(1): 67-72.

[50] JZHANG C, HOU T, CHEN J, et al. Preparation of mesoporous silica microspheres with multi-hollow cores and their application in sustained drug release. Particuology, 2010, 8(5): 447-452.

[51] CHUL O, CHUNG S, SHIN S, et al. Distribution of macropores in silica particles prepared by using multiple emulsions. Journal of Colloid and Interface Science, 2002, 254(1): 79-86.

[52] WU W, XIAO X H, ZHANG S F, et al. One-pot reaction and subsequent annealing to synthesis hollow spherical magnetite and maghemite nanocages. Nanoscale Research Letters, 2009, 4(8): 926-931.

[53] FANG X, ZHAO X, FANG W, et al.Self-templating synthesis of hollow mesoporous silica and their applications in catalysis and drug delivery. Nanoscale, 2013, 5(6): 2205-2218.
[54] WANG Q, LIU Y, YAN H. Mechanism of aself-templatingsynthesis of monodispersed hollow silica nanospheres with tunable size and shell thickness. Chemical Communications, 2007(23): 2339-2341.

[55] BAO Y, YANG Y Q, MA J Z. Research progress of hollow structural materials prepared via templating method. Journal of Inorganic Materials, 2013, 28(5): 459-468.

[56] ZHANG Q, ZHANG T, GE J, et al. Permeable silica shell through surface-protected etching. Nano letters, 2008, 8(9): 2867-2871.

[57] HU J, WANG X, LIU L, et al. A facile and general fabrication method for organic silica hollow spheres and their excellent adsorption properties for heavy metal ions. Journal of Materials Chemistry A, 2014, 2(46): 19771-19777.

[58] CHEN D, LI L, TANG F, et al. Facile and scalable synthesis of tailored silica "nanorattle" structures. Advanced Materials, 2009, 21(37): 3804-3807.

[59] CHEN Y, CHEN H R, SHI J L. Construction of homogenous/heterogeneous hollow mesoporous silica nanostructures by silica- etching chemistry: principles, synthesis, and applications. Accounts of Chemical Research, 2013, 47(1): 125-137.

[60] CHEN Y, XU P, CHEN H, et al. Colloidal HPMO nanoparticles: silica-etching chemistry tailoring, topological transformation, and nano-biomedical applications. Advanced Materials, 2013, 25(22): 3100-3105.

[61] JI Q, GUO C, YU X, et al. Flake-shell capsules: adjustable inorganic structures. Small, 2012, 8(15): 2345-2349.

[62] TERENTYEVA T G, MATRAS A, VAN ROSSOM W, et al. Bioactive flake-shell capsules: soft silica nanoparticles for efficient enzyme immobilization. Journal of Materials Chemistry B, 2013, 1(26): $3248-3256$.

[63] SLOWING I, VIVERRO E J, WU C, et al. Mesoporous silica nanoparticles as controlled release drug delivery and gene transfection carriers. Advanced Drug Delivery Reviews, 2008, 60(11): $1278-1288$.

[64] LEE C, CHENG S, HUANG I, et al. Intracellular pH-responsive mesoporous silica nanoparticles for the controlled release of anticancer chemotherapeutics. Angewandte Chemie International Edition, 2010, 122(44): 8390-8395.

[65] HWANG Y J, OH C, OH S G. Controlled release of retinol from silica particles prepared in $\mathrm{O} / \mathrm{W} / \mathrm{O}$ emulsion: The effects of surfactants and polymers. Journal of Controlled Release, 2005, 106(3): 339-349.

[66] THEISINGER S, SCHOELLER K, OSBORN B, et al. Encapsulation of a fragrance via miniemulsion polymerization for temperature-controlled release. Macromolecular Chemistry and Physics, 2009, 210(6): 411-420.

[67] VALLET-REGI M, BALAS F, COLILLA M, et al. bone-regen- 
erative bioceramic implants with drug and protein controlled delivery capability. Progress in Solid State Chemistry, 2008, 36(3): $163-191$.

[68] SHE X, CHEN L, VELLEMAN L, et al. Fabrication of high specificity hollow mesoporous silica nanoparticles assisted by Eudragit for targeted drug delivery. Journal of Colloid and Interface Science, 2015, 445: 151-160.

[69] LI Z Z, WEN L X, SHAO L, et al. Fabrication of porous hollow silica nanoparticles and their applications in drug release control. Journal of Controlled Release, 2004, 98(2): 245-254.

[70] BOTTERHUIS N E, SUN Q, MAGUSIN P C M M, et al. Hollow silica spheres with an ordered pore structure and their application in controlled release studies. Chemistry-A European Journal, 2006, 12(5): 1448-1456.

[71] CHEN J F, DING H M, WANG J X, et al. Preparation and characterization of porous hollow silica nanoparticles for drug delivery application. Biomaterials, 2004, 25(4): 723-727.

[72] HUDSON S P, PADERERA R F, LANGER R, et al. The biocompatibility of mesoporous silicates. Biomaterials, 2008, 29(30): 4045-4055.

[73] WANG J X, WANG Z H, CHEN J F, et al. Direct encapsulation of water-soluble drug into silica microcapsules for sustained release applications. Materials Research Bulletin, 2008, 43(12): 3374-3381.

[74] LANGER R. Polymer-controlled drug delivery systems. Accounts of Chemical Research, 1993, 26(10): 537-542.

[75] JONES M N. Carbohydrate-mediated liposomal targeting and drug delivery. Advanced Drug Delivery Reviews, 1994, 13(3): 215-249.

[76] LIU G, ZHU C, XU J, et al. Thermo-responsive hollow silica microgels with controlled drug release properties. Colloids and Surfaces B: Biointerfaces, 2013, 111(6): 7-14.

[77] CHEN F, ZHU Y. Chitosan enclosed mesoporous silica nanoparticles as drug nano-carriers: sensitive response to the narrow $\mathrm{pH}$ range. Microporous and Mesoporous Materials, 2012, 150(1): 83-89.
[78] XUE M, FINDENEGG G H. Lysozyme as a pH-responsive valve for the controlled release of guest molecules from mesoporous silica. Langmuir, 2012, 28(50): 17578-17584.

[79] YOU Y Z, KALEBAILA K K, BROCK S L. Temperature- controlled uptake and release in PNIPAM-modified porous silica nanoparticles. Chemistry of Materials, 2008, 20(10): 3354-3359.

[80] CHEN L, WANG W, SU B, et al. A light-responsive release platform by controlling the wetting behavior of hydrophobic surface. ACS Nano, 2014, 8(1): 744-751.

[81] CHEN M, HUANG C, HE C, et al. A glucose-responsive controlled release system using glucose oxidase-gated mesoporous silica nanocontainers. Chemical Communications, 2012, 48(76): 9522-9524.

[82] RADHAKRISHNAN K, GUPTA S, GNANADHAS D P, et al. Protamine-capped mesoporous silica nanoparticles for biologically triggered drug release. Particle \& Particle Systems Characterization, 2014, 31(4): 449-458.

[83] ZHANG Z, BALOGH D, WANG F, et al. Biocatalytic release of an anticancer drug from nucleic-acids-capped mesoporous $\mathrm{SiO}_{2}$ Using DNA or molecular biomarkers as triggering stimuli. ACS Nano, 2013, 7(10): 8455-8468.

[84] GUI W Y, WANG W Q, JIAO X Y, et al. pH-response controlled release system based on hollow mesoporous silica nanoparticles . Scientia Sinica Chimica, 2015, 45(7): 703-709.

[85] HU J, LIU L, XIE Y, et al. Facile synthesis of thermal-responsive $\mathrm{P}(\mathrm{NIPAM}-\mathrm{S}) / \mathrm{SiO}_{2}$ hybrid hollow spheres and their controllable release properties for fragrance. Polymer Chemistry, 2013, 4(11): 3293-3299.

[86] HU J, CHEN M, TIAN H, et al. Preparation and pyrolysis characteristics of PNIPAM-grafted $\mathrm{SiO}_{2}$ hollow spheres loading vitamin C. RSC Advances, 2015, 5(99): 81134-81141.

[87] ZHU Y, MENG W, GAO H, et al. Hollow mesoporous silica/poly (L-lysine) particles for codelivery of drug and gene with enzymetriggered release property. The Journal of Physical Chemistry C, 2011, 115(28): 13630-13636. 